\title{
DIAGONAL ASYMPTOTICS FOR PRODUCTS OF COMBINATORIAL CLASSES
}

\author{
MARK C. WILSON \\ To the memory of Philippe Flajolet. He has left us, but his work remains.
}

\begin{abstract}
AвSTRACT. We generalize and improve recent results by Bóna and Knopfmacher and by Banderier and Hitczenko concerning the joint distribution of the sum and number of parts in tuples of restricted compositions. Specifically, we generalize the problem to general combinatorial classes and relax the requirement that the sizes of the compositions be equal. We extend the main explicit results to enumeration problems whose counting sequences are Riordan arrays. In this framework, we give an alternative method for computing asymptotics in the supercritical case of Flajolet and Sedgewick, avoiding explicit diagonal extraction. We claim that this method is more computationally efficient.
\end{abstract}

\section{INTRODUCTION}

Let $\mathscr{S}=\left(S_{1}, \ldots, S_{d}\right)$ be a tuple of subsets of $\mathbb{N}_{+}:=\{1,2,3 \ldots\}$. By an $\mathscr{S}$-composition of $\mathbf{n} \in \mathbb{N}^{d}$ we mean a representation of $\mathbf{n}:=\left(n_{1}, \ldots, n_{d}\right)$ via ordered sums $n_{i}=\sum_{j=1}^{k_{i}} s_{i j}$ where each $s_{i j} \in S_{i}$. This is a straightforward generalization of the usual definition (when $d=1$ ) of restricted composition of a natural number, and is readily encoded by the matrix $\left(s_{i j}\right)$ once $\mathscr{S}$ is fixed. There are associated counting problems involving enumeration of $\mathscr{S}$-compositions according to size and number of parts, and these translate as usual to probabilistic questions with respect to the uniform distribution. One particular question is: what is the probability $\pi_{\mathbf{n}}$ that two $\mathbf{n}$-compositions have the same number of parts in each component? Bóna and Knopfmacher [3] studied this problem for $d=2, S_{1}=S_{2}$ and $n_{1}=n_{2}$, obtaining exact formulae for a few special choices of $S_{1}$. Banderier and Hitczenko [1] generalized the problem to the case where each $S_{i}$ is arbitrary, and from 2 to $d$-tuples, still remaining in the case $\mathbf{n}=n \mathbf{1}$ where all $n_{i}$ are equal. They obtained an explicit univariate generating function via the usual diagonal extraction method in some cases. They computed first-order asymptotics for the probability by means of a Gaussian local limit theorem essentially proved by Bóna and Flajolet [2]. This enables higher order terms to be computed in principle when combined with the diagonal extraction step, overcoming some known serious difficulties with the latter approach.

1.1. Our contribution. We generalize and improve the above results in several ways. First, we have already generalized the problem treated by previous authors by not requiring all $n_{i}$ to be equal. We generalize from compositions of integers to more general combinatorial classes. Next, we derive a generating function representation for two generalizations of the sequence construction used above, namely the functional composition schema $[6]$ and Riordan arrays. This yields a whole class of identities involving sums of squares, many of which are apparently not listed in $[\overline{8} \mid$. Turning to asymptotics in the Riordan array case, we show how to more efficiently compute full asymptotic expansions by adopting a multivariate approach, avoiding explicit diagonal extraction and using a small fragment of the theory of asymptotic multivariate coefficient extraction developed by Pemantle and Wilson [12]. This bears out the observation of Raichev and Wilson [14] that the usual diagonal

Date: March 13, 2013.

1991 Mathematics Subject Classification. 05A15, 05 A16. 
extraction method should be avoided in such problems if asymptotics are the main goal. We also deal with more general (non-Gaussian) limit results and explore the original problem of restricted compositions in more depth. We list several ideas for future work.

\section{2. $\mathscr{S}$-COMPOSITIONS}

Let $S \subseteq \mathbb{N}_{+}$and let $p(x)=\sum_{s \in S} x^{s}$. As usual, the bivariate generating function enumerating compositions with parts restricted to $S$ (identified with finite sequences of elements of $S)$ by total and number of parts is $P(x, y)=(1-y p(x))^{-1}$. Thus the $2 d$-variate generating function for $\mathscr{S}$-compositions is

$$
F(\mathbf{x}, \mathbf{y})=\frac{1}{\prod_{i=1}^{d}\left[1-y_{i} p_{i}\left(x_{i}\right)\right]} .
$$

Here $x_{i}$ marks the size and $y_{i}$ the number of parts in the $i$ th restricted composition (of $n_{i}$ using parts from $\left.S_{i}\right)$.

A pair $(\alpha, \beta)$ where each element is a restricted composition and $\alpha$ and $\beta$ have the same number of parts can be mapped bijectively to a sequence of pairs:

$$
\left(\left(\alpha_{1}, \ldots, \alpha_{k}\right),\left(\beta_{1}, \ldots, \beta_{k}\right)\right) \leftrightarrow\left(\left(\alpha_{1}, \beta_{1}\right), \ldots\left(\alpha_{k}, \beta_{k}\right)\right) .
$$

The analogous result also holds by induction for $d$-tuples for each fixed $d \geq 2$. Thus the generating function for $d$-tuples of restricted compositions each having the same number of parts, and where the $i$ th restricted set is $S_{i}$, is given by

$$
\frac{1}{1-\prod_{i=1}^{d} p_{i}\left(x_{i}\right)}:=\frac{1}{H(\mathbf{x})} .
$$

The coefficient $\left[\mathbf{x}^{\mathbf{n}}\right] H(\mathbf{x})^{-1}$ yields the number of $d$-tuples of compositions with each element having the same number of parts and the sum of the $i$ th composition being $n_{i}$. Setting all $n_{i}=n$ yields the only case studied in [3, 1 ].

\section{Generalization to combinatorial Classes}

We generalize the setup of the previous section by allowing for general weighted combinatorial classes. Let $A_{i}$ be a combinatorial class where each element $\alpha \in A_{i}$ has a nonnegative real weight $p_{i}(\alpha)$. Let $F_{i}(x, y)$ be the generating function that enumerates $A_{i}$ with respect to two integer-valued parameters $\eta_{i}, \kappa_{i}$ :

$$
F_{i}(x, y)=\sum_{n, k} a_{i ; n k} x^{n} y^{k}=\sum_{\alpha_{i} \in A_{i}} p_{i}(\alpha) x^{\eta_{i}\left(\alpha_{i}\right)} y^{\kappa_{i}\left(\alpha_{i}\right)} .
$$

Here $a_{i ; n k}$ is the total weight of the set $\left\{\alpha \in A_{i} \mid n_{1}(\alpha)=n, k_{1}(\alpha)=k\right\}$.

Example 3.1. By attaching a weight to each integer in $S$, we can consider weighted compositions. For example if $S=\{1,2\}$, and 1 and 2 have respective weights $1 / 2$ and $1 / 3$, the bivariate generating function for $S$-compositions is given by

$$
F_{i}(x, y)=\frac{1}{1-y\left(x / 2+x^{2} / 3\right)} .
$$

The compositions with 2 parts are 11,12,21,22 and these have sizes 2,3,3,4 and weight 1/4,5/6,5/6,2/3 respectively. 
The generating function

$$
\begin{aligned}
F(\mathbf{x}, \mathbf{y}) & =\prod_{i=1}^{d} F_{i}\left(x_{i}, y_{i}\right)=\sum_{\alpha \in \prod_{i} A_{i}} p_{1}\left(\alpha_{1}\right) \cdots p_{d}\left(\alpha_{d}\right) x_{1}^{\eta_{1}\left(\alpha_{1}\right)} \cdots x_{d}^{\eta_{d}\left(\alpha_{d}\right)} y_{1}^{\kappa_{1}\left(\alpha_{1}\right)} \cdots y_{d}^{\kappa_{d}\left(\alpha_{d}\right)} \\
& :=\sum_{\alpha \in \prod_{i} A_{i}} p(\alpha) \mathbf{x}^{\eta(\alpha)} \mathbf{y}^{\kappa(\alpha)}
\end{aligned}
$$

enumerates weighted $d$-tuples of elements where the $i$ th element of each tuple comes from $A_{i}, x_{i}$ marks the value of parameter $\eta_{i}$ and $y_{i}$ the value of parameter $\kappa_{i}$ on $\alpha_{i}$. Grouping terms with the same values of $\eta_{i}$ and $\kappa_{i}$ yields the representation

$$
F(\mathbf{x}, \mathbf{y})=\sum_{\mathbf{n}, \mathbf{k}} a_{\mathbf{n}, \mathbf{k}} \mathbf{x}^{\mathbf{n}} \mathbf{y}^{\mathbf{k}}
$$

where

$$
a_{\mathbf{n}, \mathbf{k}}=\prod_{i=1}^{d} a_{i ; n_{i}, k_{i}}
$$

The diagonal $\operatorname{diag}_{\mathbf{y}} F$ is the generating function derived from $F$ by restricting to the case where all $\kappa_{i}\left(\alpha_{i}\right)$ are equal:

$$
\operatorname{diag}_{\mathbf{y}} F(\mathbf{x}, t)=\sum_{\left\{\alpha \mid \kappa_{i}\left(\alpha_{i}\right)=k\right\}} p(\alpha) x_{1}^{\eta_{1}\left(\alpha_{1}\right)} \ldots x_{d}^{\eta_{d}\left(\alpha_{d}\right)} t^{k}:=\sum_{\mathbf{n}, k} b_{\mathbf{n}, k} x_{1}^{n_{1}} \ldots x_{d}^{n_{d}} t^{k}
$$

where $b_{\mathbf{n}, k}=a_{\mathbf{n}, k \mathbf{1}}$. Setting $t=1$ sums over all $k$. Then we can extract coefficients for any given choice of $\mathbf{n}=\left(n_{1}, \ldots, n_{d}\right)$ :

$$
\left[\mathbf{x}^{\mathbf{n}}\right] \operatorname{diag}_{\mathbf{y}} F(\mathbf{x}, 1)=\sum_{k \geq 0} a_{\mathbf{n}, k \mathbf{1}}=\sum_{k \geq 0} \prod_{i=1}^{d} a_{i ; n_{i}, k} .
$$

The special case of the leading diagonal $\mathbf{n}=n \mathbf{l}$ was studied in $\mid 3,1]$. The simplest special case of this occurs when all $F_{i}$ are equal, in which case we have the formula

$$
\left[\mathbf{x}^{n \mathbf{1}}\right] \operatorname{diag}_{\mathbf{y}} F(\mathbf{x}, 1)=\sum_{k \geq 0}\left(a_{n k}\right)^{d} .
$$

3.1. Special schemata. The analysis so far has been completely general. If a reasonably explicit exact formula for the coefficients is desired, we seemingly need to be able to compute $\operatorname{diag}_{\mathbf{y}} F(\mathbf{x}, 1)$ explicitly. To that end, we now restrict to the functional composition schema of [6]. Let $g(x)=\sum_{n \geq 0} a_{n} x^{n}, h(x)$ be univariate power series such that $g(0)=0$ and consider the bivariate power series $F$ given by

$$
F(x, y)=g(y h(x))=\sum_{k \geq 0} a_{k} y^{k} h(x)^{k} .
$$

Proposition 3.2. $\operatorname{Let}_{i}(x, y)=g_{i}\left(y h_{i}(x)\right)$ be as in (1). Then

$$
\operatorname{diag}_{\mathbf{y}} F\left(x_{1}, x_{2}, \ldots, x_{d}, t\right)=g_{*}\left(t h_{1}\left(x_{1}\right) \cdots h_{d}\left(x_{d}\right)\right)
$$

where $g_{*}=g_{1} * g_{2} * \cdots * g_{d}$ denotes the Hadamard product. Thus

$$
\left[\mathbf{x}^{\mathbf{n}}\right] \operatorname{diag}_{\mathbf{y}} F(\mathbf{x}, 1)=\sum_{k \geq 0} \prod_{i=1}^{d}\left[x_{i}^{n_{i}}\right] a_{i ; k} h_{i}\left(x_{i}\right)^{k}=\sum_{k \geq 0} \prod_{i=1}^{d}\left[x^{n_{i}}\right] F_{i}(x, 1) .
$$


Proof. This is a direct computation from the definitions.

$$
\begin{aligned}
\operatorname{diag}_{\mathbf{y}} F(\mathbf{x}, t) & =\sum_{\mathbf{n}, k} \mathbf{x}^{\mathbf{n}} t^{k}\left[\mathbf{x}^{\mathbf{n}} \mathbf{y}^{k \mathbf{1}}\right] F(\mathbf{x}, \mathbf{y}) \\
& =\sum_{\mathbf{n}, k} \mathbf{x}^{\mathbf{n}} t^{k}\left[\mathbf{x}^{\mathbf{n}} \mathbf{y}^{k \mathbf{1}}\right] \prod_{i} F_{i}\left(x_{i}, y_{i}\right) \\
& =\sum_{\mathbf{n}, k} \mathbf{x}^{\mathbf{n}} t^{k} \prod_{i}\left[x_{i}^{n_{i}} y_{i}^{k}\right] F_{i}\left(x_{i}, y_{i}\right) \\
& =\sum_{\mathbf{n}, k} \mathbf{x}^{\mathbf{n}} t^{k} \prod_{i} a_{i ; k}\left[x_{i}^{n_{i}}\right] h_{i}\left(x_{i}\right)^{k} \\
& =\sum_{\mathbf{n}, k} \mathbf{x}^{\mathbf{n}} t^{k} \prod_{i} a_{i ; k}\left[\mathbf{x}^{\mathbf{n}}\right]\left(\prod_{i} h_{i}\left(x_{i}\right)\right)^{k} \\
& =\sum_{\mathbf{n}, k} \prod_{i} a_{i ; k}\left(t \prod_{i} h_{i}\left(x_{i}\right)\right)^{k} \\
& =g_{*}\left(t \prod_{i} h_{i}\left(x_{i}\right)\right)
\end{aligned}
$$

Example 3.3. The special case of weighted compositions studied in [1] occurs when $g(x)=$ $1 /(1-x)$ (the sequence construction) and $h_{i}(x)=\sum_{s \in S} p_{i s} x^{s}$, with $p_{i s}>0$. Thus $g_{*}=g$. Note that [1] also requires that $\sum_{s \in S} p_{i s}>1$, but this is for convenience in asymptotic analysis, and need not be assumed yet.

In this special case we obtain

$$
\operatorname{diag}_{\mathbf{y}} F\left(x_{1}, \ldots, x_{d}, t\right)=\frac{1}{1-t \prod_{i=1}^{d} p_{i}\left(x_{i}\right)}
$$

as previously seen.

A different generalization of the sequence construction is quite useful. A Riordan array is defined by a bivariate generating function of the form

$$
F(x, y)=\phi(x) /(1-y \nu(x))
$$

where $v(0)=0$. Riordan arrays have many combinatorial and probabilistic interpretations, intimately tied to sums of independent random variables, certain types of lattice walk enumeration problems, and to Lagrange inversion. Note that Riordan arrays do not fall into the functional composition schema above - there is no way in general to write a generating function of the form (2) as $g(y h(x))$ for univariate $g, h$. Of course when $y=1$, for example, then we can express the specialized Riordan generating function in that form, with $g(x)=\tilde{\phi}(x) /(1-x)$, where $\tilde{\phi}(\nu(x))=\phi(x)$ (this is possible if $\nu^{\prime}(0) \neq 0$, in which case we have a so-called proper Riordan array).

The next result also follows by direct computation from the definitions.

Proposition 3.4. Let $F_{i}(x, y)$ generate Riordan arrays as in (2). Then

and

$$
\operatorname{diag}_{\mathbf{y}} F\left(x_{1}, x_{2}, \ldots, x_{d}, t\right)=\frac{\phi_{1}\left(x_{1}\right) \phi_{2}\left(x_{2}\right) \ldots \phi_{d}\left(x_{d}\right)}{1-t v_{1}\left(x_{1}\right) v_{2}\left(x_{2}\right) \ldots v_{d}\left(x_{d}\right)}:=\frac{\Phi(\mathbf{x})}{1-t V(\mathbf{x})}
$$

$$
\left[\mathbf{x}^{\mathbf{n}}\right] \operatorname{diag}_{\mathbf{y}} F(\mathbf{x}, 1)=\sum_{k \geq 0} \prod_{i=1}^{d}\left[x^{n_{i}}\right] \phi_{i}(x) v_{i}(x)^{k}
$$


Riordan arrays arise from many lattice path counting problems.

Example 3.5 (Dyck walks). Let $a_{n k}$ be the number of Dyck walks from the origin to $(n, k)$. Recall that a Dyck walk is a discrete walk which at each time step adds $(1,1)$ or $(1,-1)$ to its current position vector, and never leaves the positive quadrant. The generating function for the array $a_{n k}$ is of Riordan type with $v(x) / x=\phi(x)=C(x):=\left(1-\sqrt{1-4 x^{2}}\right) /\left(2 x^{2}\right)$. Thus, for example, the generating function for pairs of Dyck walks whose endpoint $y$-coordinates are equal is

$$
\frac{C\left(x_{1}\right) C\left(x_{2}\right)}{1-x_{1} x_{2} C\left(x_{1}\right) C\left(x_{2}\right)} .
$$

The numbers $a_{n k}$ are described in [8, A053131]. Note that $a_{n k}=0$ unless $n$ and $k$ have the same parity and $0 \leq k \leq n$, in which case

$$
a_{n k}=\frac{k+1}{n+1}\left(\begin{array}{c}
n+1 \\
\frac{n-k}{2}
\end{array}\right) .
$$

Dyck walks are of course identified with many other combinatorial objects. For example, the preorder traversal of a binary tree gives a bijection between Dyck walks ending at $(n, k)$ and binary trees with $n$ internal nodes and $n+1-k$ leaves. Here each internal node represents the step $(1,1)$ and each leaf the step $(1,-1)$. Thus the generating function of (3) also enumerates (by number of internal nodes) pairs of binary trees with the same number of leaves.

Example 3.6 (generalized Dyck walks). Generalizing the previous example, we consider plane walks with steps belonging to a finite set $E \subseteq \mathbb{Z}^{2}$, and constrained to lie in the upper half-plane. We call such walks constrained $E$-walks or generalized Dyck walks (the terminology meanders is used in [6]). We restrict to the case where elements of $E$ are all of the form $\left(a_{i}, b_{i}\right)$, where $a_{i}>0$ for all $i$.

Let $p=\min _{i} b_{i}$. In [4] it was shown that if $p \geq 0$ the bivariate generating function $F(x, y)$ enumerating walks by their endpoint will be rational, while otherwise it will be algebraic and irrational. We restrict further to the special case where $P:=\max b_{i}=1$. In this case $F$ is always of Riordan type where $v(x)=x \phi(x)$ (a so-called renewal array, the set of which forms the Bell subgroup), as explained in [12, Section 7.3].

Note that the bivariate generating functions for unconstrained E-walks is always rational.

If $a_{i}=1$ for all $i$, then there is a well-known interpretation in terms of walks on $\mathbb{Z}$, where the $x$-coordinate represents time and the $y$-coordinate the current position. There is an interpretation in terms of gambling - a constrained $E$-walk is a history of successive bets where the gambler starts with stake 0 and never goes bankrupt, and the step $\left(1, b_{i}\right)$ represents a payoff $b_{i}$ to the gambler. In addition to Dyck walks, another wellknown class of constrained $E$-walks of this type is that of Motzkin walks, corresponding to $E=\{(1,1),(1,-1),(1,0)\}$. Here

$$
v(x)=x \phi(x)=\frac{1-x-\sqrt{1-2 x-3 x^{2}}}{2 x} .
$$

The corresponding array is described in [8, A026300]. Schröder walks correspond to $E=$ $\{(1,1),(1,-1),(2,0)\}$. The corresponding array is described in [8, A104219].

Dyck paths are sometimes defined in terms of superdiagonal paths, namely walks which never go below the line $y=x$. The relation between the two definitions is as follows. The subgroup of $\mathbb{Z}^{2}$ generated by $(1,1)$ and $(1,-1)$ is isomorphic to $\mathbb{Z}^{2}$ via the map sending $(1,1)$ to $(0,1)$ and $(1,-1)$ to $(1,0)$. This map is the composition of rotation by $\pi / 2$ and dilation 
by $1 / \sqrt{2}$. Each Dyck walk corresponds to a superdiagonal path. For Motzkin paths, $(1,1)$ maps to $(0,1),(1,-1)$ to $(1,0)$, and hence $(1,0)$ to $(1 / 2,1 / 2)$. Schröder paths correspond to superdiagonal paths using the steps $(0,1),(1,0),(1,1)$, via the same isomorphism, which takes $(2,0)$ to $(1,1)$.

3.2. Explicit formulae. Explicit formulae for the numbers $\left[\mathbf{x}^{\mathbf{n}}\right] \operatorname{diag}_{\mathbf{y}} F(\mathbf{x}, 1)$ involving nested sums of binomials and related special sequences follow directly from the discussion above.

Example 3.7 (Binomial coefficients). Consider the Pascal triangle, generated by

$$
F(x, y)=\frac{1}{(1-x-x y)}=\frac{\phi(x)}{1-y v(x)}
$$

where $\phi(x)=1 /(1-x)$ and $v(x)=x /(1-x)$. Thus

$$
\left[\mathbf{x}^{\mathbf{n}}\right] \operatorname{diag}_{\mathbf{y}} F(\mathbf{x}, 1)=\sum_{k=0}^{n} \prod_{i=1}^{d}\left(\begin{array}{c}
n_{i} \\
k
\end{array}\right) .
$$

In the case $d=2$, the right side of (4) simplifies to $\left(\begin{array}{c}n_{1}+n_{2} \\ n_{2}\end{array}\right)$. However when $d \geq 3$, even when all $n_{i}$ are equal, to $n$ say, the sum (4) for $d \geq 3$ has no closed hypergeometric form. When $d=3$ the diagonal sum is known as the $n$th Franel number. The generating function of these generalized Franel numbers for all odd $d \geq 3$ (and likely for all even $d \geq 4$ ) is not algebraic, as explained in [1].

The nested sums arising in the formulae for $\left[\mathbf{x}^{\mathbf{n}}\right] \operatorname{diag}_{\mathbf{y}} F(\mathbf{x}, 1)$, for example the diagonal values $\sum_{k=0}^{n}\left(a_{n k}\right)^{d}$, cannot generally be simplified. The generating function, while generally D-finite, is usually not easy to compute. However when $d=2$, simplification is often possible.

Proposition 3.8. Let E be a step set defining generalized Dyck walks, such that whenever $(a, b) \in E$, also $(a,-b) \in E$. Then the number of pairs $\left(\delta_{1}, \delta_{2}\right)$ of E-walks such that $\delta_{i}$ ends at $\left(n_{i}, k_{i}\right)$ and $k_{1}=k_{2}$ is equal to the number of E-walks ending at $\left(n_{1}+n_{2}, 0\right)$.

In particular, letting $a_{n k}$ denote the number of $E$-walks ending at $(n, k)$, the identity

$$
\sum_{k \geq 0}\left(a_{n k}\right)^{2}=a_{2 n, 0}
$$

holds.

Proof. There is a bijection between pairs of $E$-walks ending at $\left(n_{i}, k\right)$ (for some unspecified $k$ ) and $E$-walks ending at $\left(n_{1}+n_{2}, 0\right)$. Given a pair as above, reverse the second by reflecting in the line $x=n_{1}$, and append it to the first, giving an $E$-walk returning to the $x$-axis at time $n_{1}+n_{2}$. Given an $E$-walk returning to the axis at time $n_{1}+n_{2}$, let $k$ be the height achieved after time $t_{1}$, split the walk at this point, and reverse the second half to obtain the second walk. The symmetry in the step set implies that the reversed walks are themselves $E$-walks of the same type.

Example 3.9. Proposition 3.8 applies in particular to Dyck, Motzkin and Schröder walks. For Dyck walks, (5) yields

$$
\sum_{\substack{0 \leq k \leq n \\
2 \mid(n-k)}}\left[\frac{k+1}{n+1}\left(\begin{array}{c}
n+1 \\
\frac{n-k}{2}
\end{array}\right)\right]^{2}=\frac{1}{n+1}\left(\begin{array}{c}
2 n \\
n
\end{array}\right) .
$$

The identity 5 leads to many explicit formulae for sums of squares, some of which appear to be new. For example (6) is mentioned in a comment by Paul D. Hanna at $[8$, A053121], but the analogous identities for Motzkin and Schröder walks do not appear to be mentioned anywhere in [8]. 
Explicit formulae for higher values of $d$ may be useful in some situations, for example when proving congruence properties. However for most applications, we should be satisfied with an asymptotic approximation, which is the subject of the next section.

\section{AsYMPtotics}

So far most of the analysis has not required any combinatorial interpretation, but from now on in order to avoid substantial difficulties and special cases we shall restrict to generating functions $F_{i}(x, y)$ that are analytic combinatorial in the sense of the following definition.

Definition 4.1. A generating function $F(\mathbf{x})$ is combinatorial if all its coefficients are nonnegative real numbers. It is analytic combinatorial if it is combinatorial and it is analytic at $\mathbf{0 .}$

Because we deal with convergent power series and not with formal series, we can relax the requirement that $h(0)=0$ in the functional composition schema, provided that the resulting composition is analytic at $x=0$ when $y=1$.

In the case of restricted compositions [1] proceeds from $\operatorname{diag}_{\mathbf{y}} F(\mathbf{x}, 1)$ by forming the diagonal with respect to $\mathbf{x}$ and setting $t=1$. The generating function

$$
D(x):=\operatorname{diag}_{\mathbf{x}, \mathbf{y}} F(x, 1):=\operatorname{diag}_{\mathbf{x}}\left[\operatorname{diag}_{y} F(\mathbf{x}, t)\right](x, 1)
$$

then encodes the desired information, with the coefficient $\left[x^{n}\right] D(x)$ being equal to the number of $d$-tuples of compositions of $n$ having the same number of parts. First order asymptotics of these coefficients were determined by a limit theorem from [2], and this helps to compute the full expansion. See Section 7 for more details.

We present an alternative method based on the multivariate asymptotic coefficient extraction program originated in $\mid 11]$ and explained in detail in $\mid[10 \mid$. In Section 7 we argue that this method is superior to the method used in [1]. For our purposes here only a small fragment of this theory is needed. We recall it here. Each fixed vector with nonzero entries $\mathbf{r}=\left(r_{1}, \ldots, r_{d}\right)$ corresponds to a minimal singularity $\mathbf{z}_{*}$ of the meromorphic function $F(\mathbf{z})=G(\mathbf{z}) / H(\mathbf{z})$ in the positive orthant, which is strictly minimal if there is no periodicity. Suppose that the singular variety given by $H(\mathbf{z})=0$ is smooth there, meaning that its gradient is nonzero.

The point $\mathbf{z}_{*}$ is the unique solution of the critical point equations which boil down to the conditions:

$$
\begin{aligned}
& H(\mathbf{z})=0 ; \\
& \frac{z_{i}}{r_{i}} \frac{\partial H}{\partial z_{i}} \text { is independent of } i .
\end{aligned}
$$

The basic smooth point asymptotic formula is

$$
a_{\mathbf{r}} \sim \mathbf{z}_{*}^{-\mathbf{r}} \sum_{k \geq 0} b_{l}\|\mathbf{r}\|^{-(d-1) / 2-l}
$$

(where we use the usual multi-index notation $\mathbf{z}^{\mathbf{r}}=\prod_{i} z_{i}^{r_{i}}$, and any fixed norm). Each coefficient $b_{l}$ is computable in terms of finitely many derivatives of $G$ and $H$ at $\mathbf{z}_{*}$. For the purposes of computation, we may break the symmetry between coordinates and have an analogous formula with $\|\mathbf{r}\|$ replaced by $r_{d}$, in which case we obtain (using $\tilde{b}_{k}$ for the coefficients)

$$
\tilde{b}_{0}=\frac{1}{\sqrt{\operatorname{det} 2 \pi \mathscr{Q}}} \frac{G\left(\mathbf{z}_{*}\right)}{z_{d} \partial H / \partial z_{d}} .
$$


Here $\mathscr{Q}$ denotes the Hessian of $\log g$ where $g$ expresses $r_{d}$ on $\mathcal{V}$ in terms of the other coordinates, and the square root must be chosen appropriately to ensure correct sign.

We let $\rho(\nu)$ denote the radius of convergence of the power series $v$, and $\tau(v)$ the onesided $\operatorname{limit}_{x \rightarrow \rho(v)_{-}} v(x)$. Recall [6. Chapter 9] that $F(x, y)=g(y h(x))$ is called supercritical if $\tau(h)>\rho(g)$. Note that in this case $g$ must have a finite radius of convergence, and then if $h$ has a minimal singularity that is a pole or logarithmic, it automatically holds.

The programme of Pemantle and Wilson deals with generating functions that are meromorphic in a polydisk containing the minimal singularity of interest. Supercritical phenomena with $g$ meromorphic can be dealt with readily by these multivariate methods because they always lead to meromorphic bivariate generating functions. However the Hadamard product of meromorphic functions need not be meromorphic, so we do not pursue asymptotics for the supercritical function schema.

In the case of supercritical arrays of Riordan type, the smooth point formulae above do apply and simplify considerably, as shown in |17|. In order to state the next result cleanly, we define the following commonly used notation, where as usual the prime ${ }^{\prime}$ denotes differentiation with respect to $x$.

$$
\begin{aligned}
& \mu_{v}(x):=\frac{x v^{\prime}(x)}{v(x)} \\
& \sigma_{v}^{2}(x):=\frac{x^{2} v^{\prime \prime}(x)}{v(x)}+\mu_{v}(x)-\mu_{v}(x)^{2} .
\end{aligned}
$$

Theorem 4.2. Let $F_{i}(x, y)$ be of Riordan type as in (2), and suppose that each $F_{i}$ is supercritical, and each $v_{i}$ is aperiodic. Then there is an asymptotic expansion of the form

$$
\left[\mathbf{x}^{\mathbf{n}}\right] \operatorname{diag}_{\mathbf{y}} F(\mathbf{x}, 1) \sim \mathbf{x}_{*}^{-\mathbf{n}} \sum_{l \geq 0} c_{l}\left(n_{d}\right)^{-(d-1) / 2-l}
$$

where $\mathbf{x}_{*}$ is chosen so that $\mu_{v_{i}}\left(x_{i}\right) / n_{i}$ is independent of $i$, and $\prod_{i} v_{i}\left(x_{i}\right)=1$.

The coefficients are algorithmically computable from the Taylor coefficients of the $\phi_{i}$ and $v_{i}$.

In the special case where all $F_{i}$ are equal and $\mathbf{n}=n \mathbf{1}$, the formulae simplify to

$$
\left[x^{n}\right] \operatorname{diag}_{\mathbf{x}, \mathbf{y}} F(x, 1) \sim c^{-n d} \sum_{l \geq 0} c_{l} n^{-(d-1) / 2-l}
$$

where $c$ is the positive root of the equation $v(c)=1$. In this case

$$
c_{0}=\frac{\phi(c)^{d}}{\sqrt{d} \mu_{v}(c)\left[2 \pi \frac{\sigma_{v}^{2}(c)}{\mu_{v}(c)}\right]^{\frac{d-1}{2}}} .
$$

Proof. The critical point equations are equivalent to the fact that $\mu_{\nu_{i}}\left(x_{i}\right) / n_{i}$ is independent of $i$ and $\prod_{i} \nu_{i}\left(x_{i}\right)=1$. The conditions on $v_{i}$ imply that their solution is a smooth strictly minimal singularity of $F$. Applying the basic smooth point asymptotic formula (7) and the leading term formula (8) yields the result. In the special case where all $F_{i}$, hence all $v_{i}$ and all $\phi_{i}$, are equal, the critical point is symmetric (all $x_{i}$ are equal, to $c$ say) and $v(c)^{d}=1$, hence $v(c)=1$. The Hessian simplifies in the symmetric case [14, Proposition 3.4] to

$$
d\left(1+\frac{c}{H_{d}}\left(H_{d d}-H_{d 1}\right)\right)^{d-1} .
$$

This further simplifies because of the simple form of $H$.

Remark. A generating function of Riordan type is supercritical if and only if $\tau(\nu)>1$ and $\rho(\phi)>c$, where $c$ is the positive solution to $v(x)=1$. Note that $\operatorname{diag}_{\mathbf{x}, \mathbf{y}} F(x, 1)$ is not in general a Riordan array. 
Theorem 4.2 has a large number of combinatorial applications. We present only a few examples, each chosen to illustrate a particular feature.

Example 4.3 (higher order terms). Applying (9) to the binomial coefficients of Example 3.7 gives

$$
\sum_{k=0}^{n}\left(\begin{array}{l}
n \\
k
\end{array}\right)^{d} \sim \sqrt{\frac{2^{d-1}}{d}} \frac{2^{d n}}{(\pi n)^{\frac{d-1}{2}}} .
$$

For each fixed value of $d$, the higher order terms can be algorithmically computed. For example, when $d=5$, we obtain (using the publicly available Sage package amg $[13]$ )

$$
\sum_{k=0}^{n}\left(\begin{array}{l}
n \\
k
\end{array}\right)^{5} \sim 32^{n} \cdot \frac{4}{25}\left(\frac{5 \sqrt{5}}{\pi^{2} n^{2}}-\frac{4 \sqrt{5}}{\pi^{2} n^{3}}\right)
$$

Higher order approximations can be useful for rather small values of $n$. For example, when $n=8$, the approximation in (11) yields (the nearest integer) 2802448277, while the exact value is 2816649826, so that the relative error in the approximation is slightly over $0.5 \%$. The relative error is $\Theta(1 / n)$ as $n \rightarrow \infty$.

Example 4.4 (off the main diagonal). Consider again binomial coefficients, but where we compute asymptotics for arbitrary directions $\mathbf{n}$. Provided that all ratios $n_{i} / n_{j}$ remain bounded, the asymptotics are uniform in the direction, and the leading term of the polynomial correction is of order $\|\mathbf{n}\|^{\frac{1-d}{2}}$. Because $v(x)=x /(1-x), \mu_{v}(x)=1 /(1-x)$ and so the constant $C$ is defined by the degree d equation $\prod_{i}\left(C n_{i}-1\right)=1$. On the main diagonal, all $n_{i}$ are equal, to $n$ say, and $C=2 / n$, so that all $x_{i}=1 / 2$. Away from the main diagonal we must resort to real root-finding in general. However some special values yield rational solutions. For example, when $d=3$ and $n_{1}=2 n_{2}=3 n_{3}$, we obtain $C n_{1}=4, C n_{2}=2, C n_{3}=4 / 3$. Thus the critical point coordinates are $x_{1}=3 / 4, x_{2}=1 / 2, x_{3}=1 / 4$. This yields

$$
\sum_{k \geq 0}\left(\begin{array}{c}
6 n \\
k
\end{array}\right)\left(\begin{array}{c}
3 n \\
k
\end{array}\right)\left(\begin{array}{c}
2 n \\
k
\end{array}\right) \sim\left(\frac{524288}{729}\right)^{n}\left[\frac{4 \sqrt{11}}{33 \pi n}-\frac{5446}{395307} \frac{\sqrt{11}}{\pi n^{2}}\right] .
$$

Example 4.5 (infinite sums). Consider the sequence $a_{n k}=2^{-k}\left(\begin{array}{c}n+k \\ k\end{array}\right)$. The bivariate generating function for this array is $(1-x-y / 2)^{-1}$. This is of Riordan type with $\phi(x)=1 /(1-x)$ and $v(x)=(1 / 2) /(1-x)$, and is not strictly speaking a Riordan array, because $v(0) \neq 0$. However, although the formulae of Section 3 do not make sense as formal power series, they do as convergent power series. The relevant smooth point has all coordinates equal to $1 / 2$, and we obtain

$$
a_{n \mathbf{1}, \mathbf{1}}=\sum_{k \geq 0} 2^{-d k}\left(\begin{array}{c}
n+k \\
k
\end{array}\right)^{d} \sim \frac{2^{d n}}{(\pi n)^{\frac{d-1}{2}}} 2 \sqrt{\frac{2^{d}}{d}}
$$

When $d=2$ the coefficient is expressible exactly in terms of Legendre polynomials, but presumably there is no closed form for $d \geq 3$.

Example 4.6 (family of binomial sums). In [9] the following family of binomial sums was studied, where $\varepsilon \in\{0,1\}$ and $a, d \in \mathbb{N}$.

$$
u_{r}^{(\varepsilon, a, d)}:=\sum_{k=0}^{r}(-1)^{\varepsilon k}\left(\begin{array}{l}
r \\
k
\end{array}\right)\left(\begin{array}{c}
a r \\
k
\end{array}\right) d^{k} .
$$

As noted in [9], these are the diagonal terms of

$$
\tilde{u}_{r s}:=\sum_{k=0}^{r}(-1)^{\varepsilon k}\left(\begin{array}{l}
r \\
k
\end{array}\right)\left(\begin{array}{c}
a s \\
k
\end{array}\right) d^{k}
$$


In [9], asymptotics for these were derived by applying methods of [12]. We can give a slightly cleaner analysis using our results above. First define the slightly simpler bivariate sequence

$$
\bar{u}_{r s}:=\sum_{k=0}^{r}\left(\begin{array}{l}
r \\
k
\end{array}\right)\left(\begin{array}{l}
s \\
k
\end{array}\right) c^{k}
$$

Setting $c=(-1)^{\varepsilon} d$ and seeking asymptotics in the direction $s=$ ar will yield the original problem.

The bivariate generating function for $\left(\begin{array}{l}r \\ k\end{array}\right)$ and $\left(\begin{array}{l}s \\ k\end{array}\right) c^{k}$ are respectively

$$
\begin{aligned}
\sum_{r, k}\left(\begin{array}{l}
r \\
k
\end{array}\right) x^{r} y^{k} & =\frac{1}{1-x-x y)}=\frac{\frac{1}{1-x}}{1-y \frac{x}{1-x}} \\
\sum_{r, k}\left(\begin{array}{l}
s \\
k
\end{array}\right)(-1)^{\varepsilon k} d^{k} x^{r} y^{k} & =\frac{1}{1-x-x c y}=\frac{\frac{1}{1-x}}{1-y \frac{c x}{1-x}}
\end{aligned}
$$

Each of these is a Riordan array. By Proposition 3.4

$$
\operatorname{diag}_{\mathbf{y}} F(\mathbf{x}, \mathbf{1})=\frac{\left(1-x_{1}\right)^{-1}\left(1-x_{2}\right)^{-1}}{1-\frac{x_{1}}{1-x_{1}} \frac{c x_{2}}{1-x_{2}}}=\frac{1}{\left(1-x_{1}\right)\left(1-x_{2}\right)-c x_{1} x_{2}}=\frac{1}{1-x_{1}-x_{2}+(1-c) x_{1} x_{2}} .
$$

For example, when $c=1$, which corresponds to $\varepsilon=0, d=1$, we are in the situation of Example 4.4, whereas when $c=2$, we have a well-known expression for the central Delannoy numbers. The trivial case $c=0$ gives all coefficients $\bar{u}_{r s}=1$ as expected.

The supercritical asymptotic result Theorem 4.2 holds provided the coefficients are nonnegative, which is guaranteed when $c \geq 1$ (so that $d \geq 1$ and $\varepsilon=0$ ). The alternating case where $c$ is a negative integer leads to multiple contributing singularities and hence periodicity. We omit further details: the positive case is very similar to a special case of Example 4.4 and will result in something similar to [9, Proposition 3] while the alternating case requires considerably more work, as seen in [9].

Several important subclasses of Riordan arrays have been studied. For each, $\phi$ is expressible in terms of $v$. For example, renewal arrays are defined by $v(x)=x \phi(x)$. This allows us to express the coefficient $c_{0}$ in (9) in terms of $v$ only. The computations required to calculate $c_{0}$ in (9) can be difficult if we insist on solving for $v$ explicitly. Even simple problems can lead to unpleasant computations with radicals. Computation is simplified in such cases by using an appropriate automorphism of the formal power series algebra. For a proper Riordan array we always have $\nu(x)=x A(\nu(x))$ for some formal power series $A$. For example, for Dyck walks $A(v)=1+v^{2}$, while for Motzkin walks $A(v)=1+v+v^{2}$.

Proposition 4.7. Let $v$ define a supercritical renewal array and let $A$ be defined by $v(x)=$ $x A(\nu(x))$. The coefficient $c_{0}$ in (9) is given by

$$
c_{0}=\left(d(2 \pi)^{d-1}\right)^{-1 / 2} \frac{\left[A(1)-A^{\prime}(1)\right]^{d}}{\sigma_{A}(1)^{d-1}} .
$$

Proof. Straightforward computations show that $\mu_{\nu}=\left(1-\mu_{A}\right)^{-1}$ and $\sigma_{v}^{2}=\sigma_{A}^{2} /\left(1-\mu_{A}\right)^{3}$. For renewal arrays, $\phi(c)=v(c) / c=c A(\nu) / c=A(\nu)$ and since $\phi(c)=1$, the result follows by algebraic simplification.

Remark. Note that applying (12) to the case of binomial coefficients, where $A(v)=1+v$, yields the same answer as in (10), after simplification.

In the case of general Riordan arrays, we can define $\phi$ implicitly via A and another function $Z$. The details are omitted, because $Z$ can be eliminated in terms of $A$ in most applications. For example, $A(t)=1+t Z(t)$ for renewal arrays. 
Note that not all our examples so far are supercritical. For example, for Dyck walks, $\tau_{v}=1$ and $v$ achieves the value 1 at $c=1 / 2$, which is on the radius of convergence of $v$. This means that the singularity structure is more complicated, and smooth point analysis does not apply. Note that (12) does not make sense in this case.

We have so far only shown supercritical examples related to simple lattice walks. The following example has quite a different character.

Example 4.8. We consider strings over the alphabet $\{1, \ldots, m\}$. Let $a_{n k}$ denote the maximum number of distinct subsequences of length $k$ that can be found in a single string of length $n$. It is known that the initial segments of length $n$ of the infinite string consisting of repeated blocks of the string $12 \cdots m$ achieve this maximum for all $k$. The bivariate generating function is of Riordan type [7] with $\phi(x)=(1-x)^{-1}$ and $v(x)=x+x^{2}+\cdots+x^{m}$. This is supercritical since $\tau_{\nu}=\infty$ and $\rho(\phi)=1>c$ where $c$ is the positive root of $\nu(x)=1$.

We leave the interpretation of the asymptotics derived from Theorem 4.2 to the reader.

Note. What about meromorphic schema? Can at least say something? See JUNK

\section{Probabilistic interpretation}

Let $X_{i n}$ denote the random variable whose probability generating function is

$$
p_{i n}(y):=\frac{\left[x^{n}\right] F_{i}(x, y)}{\left[x^{n}\right] F_{i}(x, 1)} .
$$

Then $p_{i n}$ generates the probabilities $\pi_{i n k}:=\operatorname{Pr}\left(X_{i n}=k\right)$ that a uniformly chosen element $\alpha_{i}$ of size $n$ from $A_{i}$ has $\chi_{i}\left(\alpha_{i}\right)=k$.

The probability that a uniformly randomly chosen element $\left(\alpha_{1}, \ldots, \alpha_{d}\right)$ of $\prod_{i=1}^{d} \mathscr{A}_{i}$, where $\alpha_{i}$ has size $n_{i}$ for each $i$, also satisfies $\chi_{1}\left(\alpha_{1}\right)=k_{1}, \ldots, \chi_{d}\left(\alpha_{d}\right)=k_{d}$ is given by the coefficient extraction:

$$
\frac{\left[\mathbf{x}^{\mathbf{n}} \mathbf{y}^{\mathbf{k}}\right] F(\mathbf{x}, \mathbf{y})}{\left[\mathbf{x}^{\mathbf{n}}\right] F(\mathbf{x}, \mathbf{1})}
$$

and so the probability that all $\chi_{i}\left(\alpha_{i}\right)$ are equal is

$$
\pi_{\mathbf{n}}:=\frac{\left[\mathbf{x}^{\mathbf{n}}\right] \operatorname{diag}_{\mathbf{y}} F(\mathbf{x}, \mathbf{1})}{\left[\mathbf{x}^{\mathbf{n}}\right] F(\mathbf{x}, \mathbf{1})}=\frac{\left[\mathbf{x}^{\mathbf{n}}\right] \operatorname{diag}_{\mathbf{y}} F(\mathbf{x}, \mathbf{1})}{\prod_{i=1}^{d}\left[x^{n_{i}}\right] F_{i}(x, 1)} .
$$

In the special case where all $F_{i}$ are equal and we are concerned with the main diagonal $\mathbf{n}=n \mathbf{1}$, the formula becomes even simpler. The probability is given by

$$
\pi_{n \mathbf{1}}=\frac{\sum_{k \geq 0}\left(a_{n k}\right)^{d}}{\left[\sum_{k \geq 0} a_{n k}\right]^{d}}=\sum_{k \geq 0}\left[\operatorname{Pr}\left(X_{n}=k\right)\right]^{d}
$$

where $X_{n}$ is the random variable such that $\operatorname{Pr}\left(X_{n}=k\right)=a_{n k} / \sum_{k \geq 0} a_{n k}$, in other words the value of $\kappa$ conditional on all $\eta_{i}$ having value $n$.

In [2] the following result appears, which gives the leading term for much more general problems.

Theorem $5.1(\mid \sqrt{2 \mid})$. Suppose that $\left(X_{n}-\mu_{n}\right) / \sigma_{n}$ obeys a continuous local limit law with den$\operatorname{sity} g(x)$. Then $\pi_{n \mathbf{1}} \sim K /\left(\sigma_{n}\right)^{d-1}$ where

$$
K=\int_{-\infty}^{\infty} g(x)^{d} d x
$$




\begin{tabular}{c|c}
$d$ & $P(d)$ \\
\hline 1 & 1 \\
2 & 0.308508322553671 \\
3 & 0.106031646409446 \\
4 & 0.0377901929386580 \\
5 & 0.0136873221938897
\end{tabular}

TABLE 1. Values of $P(d)$ in Example 5.4

The result is unsurprising given (14), as the sum $\operatorname{Pr}\left(X_{n}=k\right)^{d}$ is readily approximated by the integral of $g^{d}$, normalized by an appropriate power of $\sigma_{n}$. Since a wide variety of problems lead to the Gaussian distribution, for which the constant $K$ can be explicitly evaluated, this result is rather useful. However, probabilistic methods typically only give access to the leading term asymptotic, and for higher order terms we must try elsewhere.

Note that convergence to a Gaussian limit law typically occurs for the supercritical schema (not always, as claimed in [6, Proposition IX.6], because the "variability condition" on variance being nonzero is not always met). It also occurs in other types of problems. For example, it is applicable to the case of Dyck walks, by [6, Theorem IX.12].

In the special case of supercritical arrays of Riordan type, we have the following explicit formula for $K$.

Proposition 5.2. Let $F(x, y)=\phi(x) /(1-y v(x))$ be supercritical of Riordan type. Then in (15)

$$
K=\frac{1}{\sqrt{d}}\left(\frac{\mu_{v}(c)^{3}}{2 \pi \sigma_{v}^{2}(c)}\right)^{\frac{d-1}{2}}
$$

Proof. In the supercritical case, we have convergence to a Gaussian limit. It is unnecessary to compute the constant via the integral in (15), because the denominator of (13) is simply the $d$ th power of $\left[x^{n}\right] \phi(x) /(1-v(x))$. That coefficient is asymptotically computed by the usual univariate residue approach, since there is a simple minimal pole at $c$.

Example 5.3. Applying (16) to the special case of restricted compositions considered in [1] immediately yields Theorem 5.3 of that paper. Here $v_{i}=p_{i}, v_{i}(0)=0$ and $\phi_{i}=1$, and supercriticality follows from the assumption $\sum_{x} p_{i x}>1$.

Note that the formula for $K$ in (16) is equivalent to that of [1, Theorem 5.3]. In particular, it is independent of $\phi$.

An obvious question to ask is: what happens if there is no convergence to a continuous limit law? Consider the case where there is a discrete limit law. In that case $\operatorname{Pr}\left(X_{n}=k\right)$ converges uniformly to $\operatorname{Pr}(X=k)$ for some discrete random variable $X$, and so $\operatorname{Pr}\left(X_{n}=k\right)^{d}$ converges to $\operatorname{Pr}(X=k)^{d}$.

Example 5.4. Let $X_{n}$ be the number of fixed points of a uniformly chosen permutation of size $n$. Then $X_{n} \rightarrow X$ where $X$ is Poisson with rate 1 . Thus for each $d$, the probability that each of $d$ uniformly chosen permutations of size $n$ has the same number of fixed points is approximately

$$
P(d):=e^{-d} \sum_{k \geq 0} \frac{1}{(k !) d} .
$$

When $d=2$, this is easily expressed in terms of values of a Bessel function, but, as expected, for $d>2$ there appears to be no simple expression for the sum. Table 1 gives some approximate values. The entries for $d \geq 3$ were not found in a search of the Inverse Symbolic Calculator [16]. 


\begin{tabular}{c|c}
$d$ & $P(d)$ \\
\hline 1 & 1 \\
2 & 0.185185 \\
3 & 0.040400 \\
4 & 0.009335 \\
5 & 0.002219
\end{tabular}

TABLE 2. Values of $P(d)$ in Example 6.1

\section{RESTRICTED COMPOSITIONS IN OTHER CASES}

We have seen that cases of the original composition problem that fall into the supercritical sequence schema lead to Gaussian asymptotics for which we have complete information. In this section, for completeness, we examine the other cases.

Example 6.1. First suppose that $\tau_{i}:=\sum_{s \in S_{i}} p_{i s}<1$ for all $i$. In this case the generating functions $F_{i}\left(x_{i}, y_{i}\right)$ each fall into the subcritical case. Each obeys a discrete limit law as described in [6, Chapter IX.2]. Fix $i$, let $\tau=\tau_{i}$, and introduce $X_{n}$ as above. According to [6, Proposition IX.2], the limit $X$ has probability generating function given by

$$
p(u)=\frac{u(1-\tau)^{2}}{(1-\tau u)^{2}}
$$

In other words, $\operatorname{Pr}(X=k)=k \tau^{k-1}(1-\tau)^{2}$.

The probability that d randomly chosen compositions have the same number of parts is then

$$
(1-\tau)^{2 d} \sum_{k \geq 0} k^{d}\left(\tau^{d}\right)^{k-1}
$$

This is expressible in terms of the polylogarithm as

$$
\frac{(1-\tau)^{2 d}}{\tau^{d}} \mathrm{Li}_{-d}\left(\tau^{d}\right)
$$

There are several known explicit formulas, involving Stirling numbers or Eulerian numbers, for the polylogarithm with negative integer argument. For example, it is known that $\mathrm{Li}_{-d}(x)$ has the form $(1-x)^{-(d+1)}$ times a monic polynomial in $x$ of degree $d-1$.

Table 2 gives values of the probability $P(d)=2^{-d} \mathrm{Li}_{-d}\left(2^{-d}\right)$ for small values of $d$, for the special value $\tau=1 / 2$.

In the critical case where $\sum_{s} p_{i s}=1$, we are dealing with a probability distribution on $\mathbb{N}_{+}{ }^{* * *}$

\section{CONCLUSIONS}

7.1. Comparison with other work. Our explicit results are more general than those in [1] because of the extension to arrays of Riordan type, which allows for many more applications.

Another important purpose of the present article is to make the case that the asymptotic methodology used here is superior to that used in [1], and many other papers in recent literature. We now explain this in more detail.

If $F$ is $D$-finite, then the function $D(x)=\operatorname{diag}_{\mathbf{x}, \mathbf{y}} F(x, 1)$ is D-finite, meaning that it satisfies a linear differential equation with polynomial coefficients. However, no simpler representation is available in general, even if $F$ is for example, a rational function. In order to extract asymptotics of coefficients of $D$, a method such as that of Frobenius (applied to the ODE) or Birkhoff-Trjitinsky (applied to the equivalent polynomial recursion) is often 
used (for example in [1]. Unfortunately these methods suffer from the difficulty of solving the connection problem, which means that the asymptotic scale can be determined but no rigorous method is known for determining the coefficients (some progress in the general case has been advertised by Banderier, Chern and Hwang, but nothing has been made public). In $|1|$ the coefficients were claimed to be determined by means of a local limit theorem of $|2|$, described below. Our methods allow rigorous computation of terms of every order, as explained in detail in [15].

In addition to the problems of computability, there are problems of computational complexity. The current methods of computing the defining ODE for $D(x)$ are computationally intensive and the work involved increases substantially with $d$, as noted in [1]. The alternative method presented here does not suffer from this problem. However, once we move to extract asymptotics, since the diagonal method only requires asymptotics of a 1-variable function, it is likely to be quicker than the multivariate method, which requires multivariate asymptotic extraction. No formal comparison of the overall complexity of the two entire procedures has been made. Since both involve computer algebra algorithms that have a bad worst case, the question requires further study.

Furthermore, as shown in [14], even when $D(x)$ is available, it is much more computationally intensive to perform the above procedure for multi-indices $\mathbf{n}$ away from the main diagonal $n \mathbf{1}$, and approximations that are uniform in the direction are not available. This is serious deficiency, because off-diagonal multi-indices of interest typically do not lie on a ray determined by a direction with integer coordinates.

Our methods sidestep these problems by dealing with $\operatorname{diag}_{\mathbf{y}} F$ directly (assuming that this is meromorphic) and provide explicit approximations that are uniform in direction and do not become substantially harder to compute as we move off the main diagonal, showing that for multivariate asymptotic coefficient extraction problems of this sort, the diagonal extraction method should be replaced by the methods described in |14|.

7.2. Possible extensions. Since for the purposes of asymptotics it is not necessary to compute the iterated diagonal $\operatorname{diag}_{\mathbf{x}} \operatorname{diag}_{\mathbf{y}} F$, perhaps it is not even necessary to compute $\operatorname{diag}_{\mathbf{y}} F$. However it seems to extract the information required directly from $F$. The difficulty is that the multivariate methodology described above derives asymptotics that are uniform in the central regime, where all components of $\mathbf{r}=(\mathbf{n}, \mathbf{k})$ are of the same asymptotic order (in other words, $n_{i} /\|\mathbf{r}\|$ and $k_{i} /\|\mathbf{r}\|$ are each bounded above and below by nonzero constants). The diagonal $\operatorname{diag}_{\mathbf{y}} F$ involves a sum over all values of $k$, some of which are very small. An argument that cuts off the tails of the distribution may work, but this seems more in spirit with a purely probabilistic approach, and we do not pursue it here.

The singularity analysis of Hadamard products was investigated in detail by Fill, Flajolet and Kapur [5]. This goes far beyond the meromorphic asymptotics of the present work, and rests on the theory pioneered by Flajolet and Odlyzko [6, Chapter VI]. The expression for $\operatorname{diag}_{\mathbf{y}} F(\mathbf{x}, \mathbf{1})$ derived in Proposition 3.2 should allow derivation of asymptotics in many important cases. We do not pursue it here for reasons of length and coherence of the present article.

The expression for $\pi_{n 1}$ in Theorem 5.1 should generalize in a fairly straightforward way to the off-diagonal case, provided that $\mathbf{n}$ is suitably restricted. Also, there is no need to restrict to the case where all the combinatorial classes coincide. Again, this would take us too far afield in the present work. 


\section{REFERENCES}

[1] Cyril Banderier and Paweł Hitczenko. "Enumeration and asymptotics of restricted compositions having the same number of parts". Discrete Appl. Math. 160.18 (2012), 2542-2554.

[2] Miklós Bóna and Philippe Flajolet. "Isomorphism and symmetries in random phylogenetic trees”. J. Appl. Probab. 46.4 (2009), 1005-1019.

[3] Miklós Bóna and Arnold Knopfmacher. "On the probability that certain compositions have the same number of parts". Ann. Comb. 14.3 (2010), 291-306.

[4] Mireille Bousquet-Mélou and Marko Petkovšek. "Linear recurrences with constant coefficients: the multivariate case”. Discrete Math. 225.1-3 (2000). Formal power series and algebraic combinatorics (Toronto, ON, 1998), 51-75.

[5] James A. Fill, Philippe Flajolet, and Nevin Kapur. "Singularity Analysis, Hadamard Products, and Tree Recurrences”. J. Comput. Appl. Math. 174 (2005), 271-313.

[6] Phillipe Flajolet and Robert Sedgewick. Analytic combinatorics. Cambridge University Press, 2009, p. 824.

[7] Abraham Flaxman, Aram W. Harrow, and Gregory B. Sorkin. "Strings with maximally many distinct subsequences and substrings". Electron. J. Combin. 11.1 (2004), Research Paper 8, 10 pp. (electronic).

[8] OEIS Foundation. Online Encyclopedia of Integer Sequences. 2013. URL: http : / / oeis.org.

[9] Rob Noble. "Asymptotics of a family of binomial sums". J. Number Theory 130.11 (2010), 2561-2585.

[10] Robin Pemantle and Mark C. Wilson. "Analytic Combinatorics in Several Variables" (2013).

[11] Robin Pemantle and Mark C. Wilson. "Asymptotics of multivariate sequences. I. Smooth points of the singular variety”. J. Combin. Theory Ser. A 97.1 (2002), 129-161.

[12] Robin Pemantle and Mark C. Wilson. "Twenty combinatorial examples of asymptotics derived from multivariate generating functions". SIAM Rev. 50.2 (2008), 199272.

[13] Alexander Raichev. amgf-0.8. 2013. URL:http://www . alexraichev.org/research. html.

[14] Alexander Raichev and Mark C. Wilson. "A new method for computing asymptotics of diagonal coefficients of multivariate generating functions”. In: 2007 Conference on Analysis of Algorithms, AofA 07. Discrete Math. Theor. Comput. Sci. Proc., AH. Assoc. Discrete Math. Theor. Comput. Sci., Nancy, 2007, 439-449.

[15] Alexander Raichev and Mark C. Wilson. "Asymptotics of coefficients of multivariate generating functions: improvements for smooth points”. Electron. J. Combin. 15.1 (2008), Research Paper 89, 17.

[16] Nathan Singer et al. Inverse Symbolic Calculator. 2013. URL: http: // isc2 . carma. newcastle.edu.au/advancedCalc.

[17] Mark C. Wilson. “Asymptotics for generalized Riordan arrays”. In: 2005 International Conference on Analysis of Algorithms. Discrete Math. Theor. Comput. Sci. Proc., AD. Assoc. Discrete Math. Theor. Comput. Sci., Nancy, 2005, 323-333 (electronic). 